\title{
THE
}

UNIVERSITY

University of Rhode Island

OF RHODE ISLAND

DigitalCommons@URI

12-17-1984

\section{Structure of Charged Argon Clusters Formed in a Free Jet Expansion}

\author{
I. A. Harris \\ R. S. Kidwell \\ J. A. Northby \\ University of Rhode Island, jnorthby@uri.edu
}

Follow this and additional works at: https://digitalcommons.uri.edu/phys_facpubs

Terms of Use

All rights reserved under copyright.

\section{Citation/Publisher Attribution}

Harris, I. A., Kidwell, R. S., \& Northby, J. A. (1984). Structure of Charged Argon Clusters Formed in a Free Jet Expansion. Physical Review Letters, 53(25), 2390.

Available at: http://dx.doi.org/10.1103/PhysRevLett.53.2390

This Article is brought to you for free and open access by the Physics at DigitalCommons@URI. It has been accepted for inclusion in Physics Faculty Publications by an authorized administrator of DigitalCommons@URI. For more information, please contact digitalcommons-group@uri.edu. 


\title{
Structure of Charged Argon Clusters Formed in a Free Jet Expansion
}

\author{
I. A. Harris, R. S. Kidwell, and J. A. Northby \\ Physics Department, University of Rhode Island, Kingston, Rhode Island 02881 \\ (Received 30 July 1984)
}

\begin{abstract}
We describe measurements of the mass spectrum of charged argon clusters generated in a low-temperature free jet expansion. It contains detailed intensity variations which can be understood in terms of a simplified competing-lattice model of charged-cluster structure.
\end{abstract}

PACS numbers: $36.40 .+\mathrm{d}, 61.50 . \mathrm{Cj}, 68.20 .+\mathrm{t}$

The question of how macroscopic properties such as crystal structure evolve as the number of atoms in a system increases has long been of interest. Particular attention has been paid to rare-gas systems where the interactions are both weak and relatively well understood. Theoretical modeling ${ }^{1,2}$ of such many-body systems became possible with the advent of large computers, but experimental systems with which to test predictions have been difficult to devise. Recently, the ability to produce rare-gas clusters in free jet expansions has provided such an opportunity, but not without difficulties in interpretation. For example, in experiments performed directly on neutral clusters, ${ }^{2}$ the mass is not a well defined variable. On the other hand, experiments which measure the resolved mass spectrum direct$1 y^{3-5}$ and attempt to link observed regularities ("magic numbers") to structural properties of clusters also suffer from a significant ambiguity. In order to determine the mass the clusters are first ionized, usually by electron bombardment, and the resulting charged-cluster spectrum is measured. It is likely, however, that significant fragmentation occurs in the ionization process. In this case, in addition to structure in the spectrum arising from the initial neutral-cluster distribution, additional regularities may be introduced by a mass-dependent ionization cross section, by mass-dependent energy deposition, and by variation in the stability of the resulting charged clusters.

We describe below a different experimental process, the growth of clusters on ions entrained with the jet, ${ }^{6,7}$ which does not suffer from such ambiguities in interpretation, and we report the first well resolved rare-gas mass spectrum generated in this system. Furthermore, we will show that the well defined regularities we observe arise from structure in the binding-energy curve of charged clusters, and may be understood in considerable detail in terms of a surprisingly simple model of charged-cluster structure.

In our experiment ${ }^{8}$ pure argon gas initially at 77 $\mathrm{K}$ and 70 Torr expands through a small nozzle into a vacuum. As it accelerates it cools adiabatically and becomes supersaturated. Positive ions, produced upstream from the nozzle by a corona discharge, are swept into the jet where they become condensation nuclei and clusters grow about them. Since incident gas atoms will be mostly absorbed, the heat of condensation must be carried off principally by evaporating atoms. The formation of clusters can then be described in terms of a competition between a process of accretion and heating, and a process of evaporation and cooling. ${ }^{9}$ After the gas density becomes so low that collisions effectively cease, evaporation and cooling will continue until the charged cluster reaches a bound state. It is reasonable to presume that the cluster will be more likely to end up in a tightly bound final state than a loosely bound one, and thus the mass spectrum should reflect the binding curve of charged clusters. The cluster ions produced are skimmed from the central portion of the jet, accelerated, and passed through additional stages of differential pumping into a magnetic mass spectrometer. The resulting mass spectrum from $N=10$ to 90 argon masses is shown in Fig. 1. We have identified those peaks with large values of the intensity ratio $I(N)$ / $I(N+1)$ which presumably are more tightly bound than their neighbors. We have also obtained lower-resolution spectra out to $N \sim 170$ which indicate that comparable structure persists to much larger cluster sizes, but at present our mass calibration is less certain in this range. We do see particularly significant current drops near $N \sim 115$ and $N \sim 147$, however. It is interesting to compare these results with those found by others for neutral argon jet expansions. ${ }^{4}$ There they find strong peaks at $N=14,16,19,21,23$, and 27 . This is significantly different from the sequence 13, 19, 23, 26, 29,32 , and 34 which we find in the same region and indicates that their spectrum is determined by more than just the charged-cluster stability curve. Whether it in fact represents the neutral-cluster curve as they suggest is much less certain. The most extensive neutral-cluster spectra have been measured in Xe jets, ${ }^{3}$ where strong peaks or edges were found at $N=13,19,25,55,71,81,87,135$, 


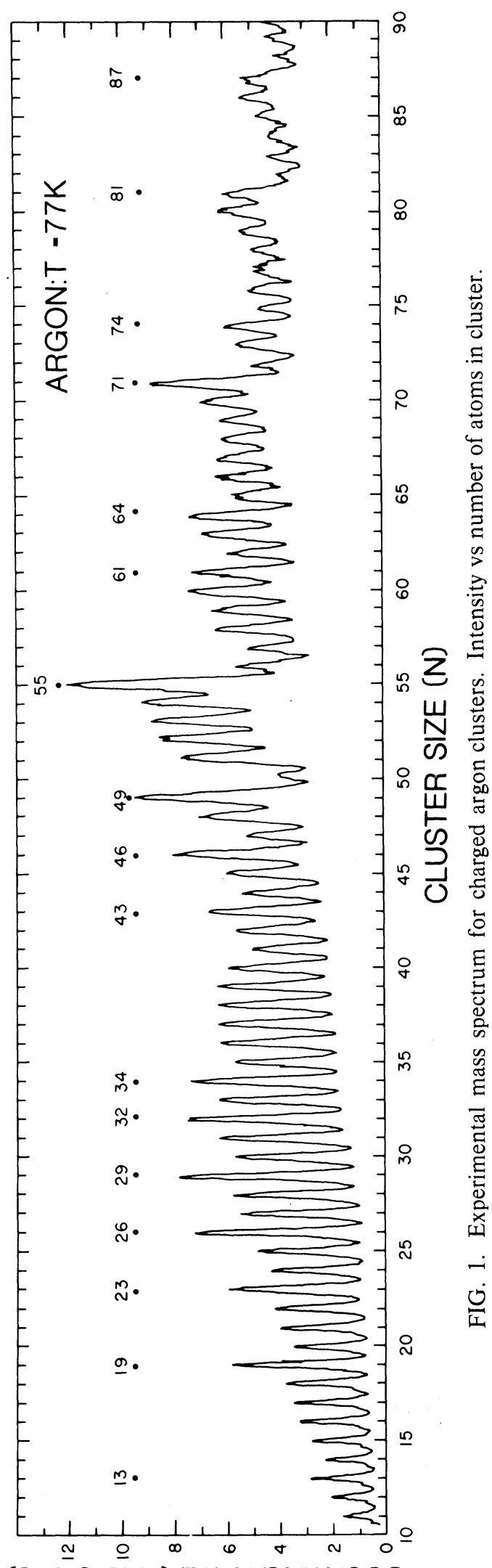

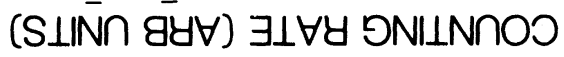

and 147. While our spectrum is richer in detail, with the exception of the $N=25 / 26$ peaks the correspondence is quite striking.

We can understand our results in more detail if we adopt the following semiquantitative model. We presume that the structure in the mass distribution becomes set in the evaporation process after accretion in the jet ceases. At this point we can describe the distribution of relatively hot clusters by $n(M, E)$, the number of $M$-atom clusters per unit volume with total energy in the range $d E$ around $E$. We assume that in a time short in comparison to the $2 \times 10^{-4}$ sec drift time the clusters will evaporate and cool until they reach a bound state. ${ }^{9}$ If we neglect the residual kinetic energy of the evaporated atoms, then every cluster with $-E_{b}(N)$ $\leqslant E \leqslant-E_{b}(N-1)$, where $E_{b}(N)$ is the binding energy of an $N$-atom cluster, will end up as a bound $\mathrm{N}$-atom cluster. Thus the final number of $\mathrm{N}$-atom clusters per unit volume is given by $I_{0}(N)$, where

$$
I_{0}(N)=\sum_{M=N}^{\infty} \int_{-E_{b}(N)}^{-E_{b}(N-1)} n(M, E) d E .
$$

If we assume further that $n(M, E)$ is slowly varying, we can remove it from the integral and obtain

$$
I_{0}(N)=\Delta E_{b}(N) \sum_{M=N}^{\infty} n\left(M, E_{b}(N)\right),
$$

where $\Delta E_{b} \equiv E_{b}(N)-E_{b}(N-1)$, and the sum is a slowly varying function of $N$ determined by conditions in the jet. In this model, then, the fine structure of the mass spectrum is nearly proportional to the successive binding-energy differences.

To our knowledge, at present there are no detailed calculations of the binding energy of charged clusters in our size range, although considerable relevant work has been done with neutrals. ${ }^{1,2,10}$ In what follows we will show that most of the observed fine structure can be understood in terms of a highly simplified model of charged-cluster structure involving only near-neighbor bond counting on lattices. We start with the observation that the strong peaks at 13,55 , and 147 probably represent the closure of the $N^{\prime}=1,2$, and 3 shells in an icosahedral packing geometry. ${ }^{1-3}$ Let us focus on the structure within the $N^{\prime}=2$ shell. For present purposes we will assume that the core structure is a rigid thirteen-atom molecular ion with icosahedral symmetry. The second shell is formed by decorating this core with atoms at sites of high symmetry until, with 42 atoms forming an icosahedral lattice, the shell is completed. There are three types of sites which are likely locations: those centered on faces (FC sites) with three near neighbors in the core, 
those centered on edges (EC sites) with two, and those centered on vertices ( $\mathrm{V}$ sites) with one. Occupation of an FC site precludes occupation of adjacent EC sites and vice versa, but occupation of a $\mathrm{V}$ site is compatible with either. Consequently there are two mutually exclusive lattices which can be supported by the core: the "FCV' lattice consisting of twenty FC and twelve $\mathrm{V}$ sites, and the "ECV" or "icosahedral" lattice consisting of thirty $\mathrm{EC}$ and twelve $\mathrm{V}$ sites. These lattices are represented schematically in Fig. 2, where $\mathrm{V}$ sites are indicated by heavy circles. We expect that the icosahedral lattice will represent the favored structure when the shell is nearly complete because it contains more sites, but that the FCV lattice will be favored when the shell is fairly empty since it optimizes intershell interactions. Our problem is to determine the minimum energy configuration when a given number of atoms is distributed on each of these lattices. Since the pair potential is short ranged, to a lowest order of approximation we will take the intrashell interaction energy to be simply proportional to the number of near-neighbor bonds. The interaction of an atom with the core is more difficult to estimate, since in addition to a modified interatomic pair potential there is also a relatively weaker polarization interaction with the central charge. ${ }^{11}$
The dominant contribution is undoubtedly the interatomic pair potential, however, and consequently we approximate again the interaction energy at a site to be proportional to the number of intershell near-neighbor bonds. At this level of approximation then, the only effect of the charge remaining is the stabilization of the thirteen-atom core structure.

To find the maximum number of bonds for a given number of atoms on a lattice we use a simple calculational scheme. Atoms are distributed at random on the lattice and then moved one at a time whenever doing so increases the number of bonds. When no more moves are possible the number of bonds and the configuration are recorded. The process is repeated many times and the binding energy is taken to be proportional to the largest number found. At least a hundred and in some cases several hundred trials were made, but in every case the largest value was found more than once in the first ten trials. In Fig. 2 the successive differences $\Delta E_{b}(N)$ obtained are given in near-neighbor bond units for each lattice. As a particular example, if atoms are removed from a filled icosahedral lattice in the sequence indicated in Fig. 2(b), the resulting configurations will maximize the number of bonds. In general we find that the tightly bound configurations at $N=49,46,43$, and 39 correspond, respec-

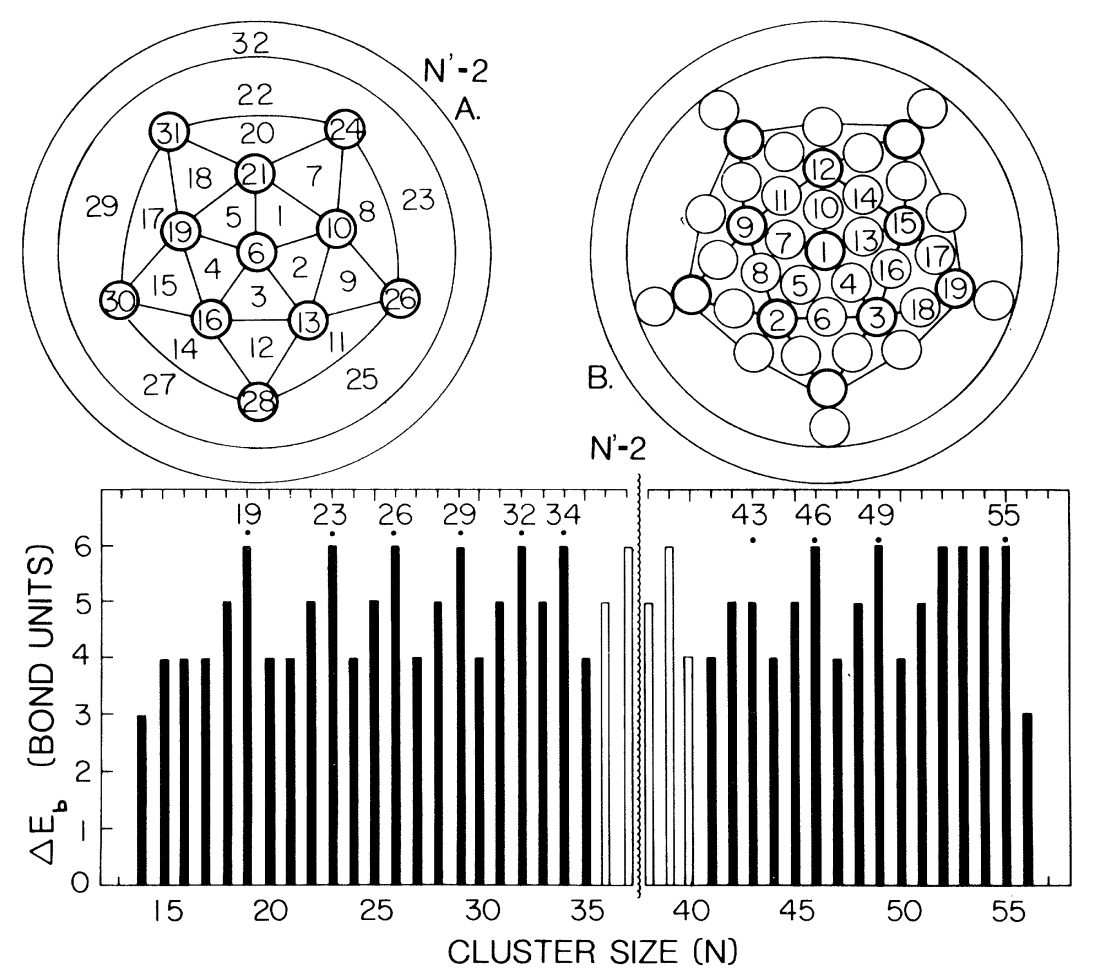

FIG. 2. Lattice diagrams and calculated binding-energy differences for (a) FCV and (b) icosahedral lattices. Heavy circles represent sites above vertices of the underyling icosahedral core. 
tively, to the removal of one, two, and three adjacent faces and the five faces forming a cap. The first three of these peaks corresponds well to prominent peaks in the observed spectrum, but for $N \lesssim 40$ the correspondence breaks down as one might expect. On the other hand, if atoms are added to the FCV lattice in the typical sequence indicated in Fig. 2(a), the resulting configurations will optimize the bond number for that lattice. In this particular scheme, atoms are placed at FC sites until they form a five-membered ring, and then the enclosed vertex site is filled forming a cap. Next an adjacent five-membered ring is completed and its enclosed vertex is filled and so forth. In general we find that the tightly bound configurations at 19,23 , $26,29,32$, and 34 correspond to the completion of one, two, three, four, five, and six adjacent caps. These correspond exactly to strong peaks in the experimental spectrum, but for $N \geq 35$ the correspondence again breaks down. Apparently the cross over between the two lattice structures occurs somewhere between 35 and 42 masses. The crossover point will depend on the absolute energy, but since the model gives only a quantity proportional to energy differences within a lattice sequence we cannot predict it. It is interesting to note, however, that with the single exception of the peak at $N=49$, the predicted peak ratios $I\left(N_{p}\right) / I\left(N_{p}+1\right)$ agree to within about $\pm 15 \%$ with the experimental ratios at each peak $N_{p}$. Thus despite its extreme simplifications the model not only describes the location of all the structural features of the observed spectrum, but also provides a reasonable estimate of their magnitude.

We can also supply arguments identical to those presented above to generate sphere-packing models for neutral clusters built on a rigid thirteen-atom icosahedral core. In this case we predict that at the level of accuracy obtained by counting nearneighbor bonds, the binding-energy differences $\Delta E_{b}(N)$ in the second shell will be the same for neutral as for charged clusters. Improved accuracy can be had, for example, by use of complete pair potentials and a more accurate core-ion interaction, and by allowance of the clusters to relax to lowerenergy deformed configurations. Clearly, as more detail is included the binding curves will change and differences between the charged and neutral cases will develop. However, we believe that the qualitative structure in $\Delta E_{b}(N)$ will remain the same in both cases, since it apparently is determined principally by the number of near neighbors in a particular configuration. This essentially geometric property is unlikely to change in the relaxation process if the starting configuration is reasonable. ${ }^{12}$

In conclusion, we want to emphasize the fact that interpretation of the experimental mass spectrum in terms of structural models is much less ambiguous in the present case when clusters are "born ionized" than when they are "born neutral" and subsequently ionized for detection. While structural modeling is clearly more difficult for charged clusters than for neutrals, the good agreement between our simplified model and the experimental spectrum indicates that outside the first coordination shell the effect of the charge core is not large. Consequently, we believe that the charge-cluster system provides the best available means of experimentally testing calculational methods for finite many-body systems, and as such it deserves more theoretical attention.

We gratefully acknowledge helpful discussions with Professor L. Kahn and Professor D. Freeman. This work has been supported by the National Science Foundation through Grants No. DMR 8024366 and No. DMR 8405190.

1M. R. Hoare, Adv. Chem. Phys. 40, 49 (1979).

2J. Farges et al., J. Chem. Phys. 78, 5067 (1983), and 59, 3454 (1973).

${ }^{3}$ O. Echt, K. Sattler, and E. Recknagel, Phys. Rev. Lett. 47, 1121 (1981).

${ }^{4}$ A. Ding and J. Hesslich, Chem. Phys. Lett. 94, 54 (1983)

5P. W. Stephens and J. G. King, Phys. Rev. Lett. 51, 1538 (1983).

6J. Q. Searcy and J. B. Fenn, J. Chem. Phys. 61, 5282 (1974).

${ }^{7}$ L. Friedman and R. J. Beuhler, J. Chem. Phys. 78, 4669 (1983).

8I. A. Harris, R. S. Kidwell, and J. A. Northby, in Proceedings of the Seventeenth International Conference on Low Temperature Physics, edited by U. Eckern, A. Schmid, W. Weber, and H. Wühl (North-Holland, Amsterdam, 1984).

9J. M. Soler and N. Garcia, Phys. Rev. A 27, 3307 (1983)

${ }^{10}$ G. L. Griffin and R. P. Andres, J. Chem. Phys. 71, 2522 (1979).

${ }^{11}$ On the assumption of a central charge, the induced dipole interaction in the $N^{\prime}=2$ shell is about half the Lennard-Jones well depth and the variation among sites even less. The principal effect will be a small, relatively constant offset in $\Delta E_{b}(N)$ which we will neglect.

12J. Farges, M. F. deFeraudy, B. Raoult, and G. Torchet [private communication] have recently performed a relaxation calculation for neutral clusters starting from an FCV growth sequence similar to that generated here. They obtain the same sequence of peaks with amplitudes comparable to ours. 